\title{
Fibrin films: overlooked hemostatic barriers against microbial infiltration
}

\author{
Sean X. Gu and Steven R. Lentz \\ Department of Internal Medicine, University of lowa Carver College of Medicine, lowa City, lowa, USA.
}

\begin{abstract}
The hemostatic response to vascular injury culminates in a fibrin clot network that forms an initial barrier to blood loss and also contributes to microbial host defense. Fibrinogen is cleaved by thrombin into fibrin monomers that spontaneously polymerize into protofibrils and form the extensive fiber networks characteristic of blood clots. In this issue of the $\mathrm{JCl}$, Macrae and colleagues characterize an alternative fibrin structure in which fibrinogen and fibrin assemble into a continuous $2 \mathrm{D}$ film at the exterior face of the fibrin clot network. Fibrin films connect to the underlying fiber network through tethering fibers and provide a protective barrier to microbial infiltration. These findings shed new light on a previously overlooked mechanism of fibrin assembly at the clot surface and provide a link between hemostasis and innate immunity.
\end{abstract}

\section{Fibrin and host defense}

Blood coagulation and innate immunity share a common evolutionary ancestry as systems of rapid response to physical injury. Physical wounds not only present an immediate danger of blood loss but also open a port of entry for pathogenic microbes (1). In mammals, there is extensive molecular crosstalk between coagulation and inflammation that can either amplify or dampen host responses (2, 3). This interplay between hemostasis and innate immune defense has been termed "immunothrombosis" (1). The central components of immunothrombosis include formation of the fibrin clot matrix and activation of monocytes, neutrophils, and platelets, which together contribute to pathogen recognition, compartmentalization, and trapping. Additional features include the local release of platelet polyphosphates and neutrophil extracellular traps, leading to contact activation of coagulation and the kallikreinkinin system, with release of bradykinin and antimicrobial peptides $(4,5)$.
The fibrin clot network, in particular, has been proposed to play an important role in host antimicrobial defense $(6,7)$. In the final stage of the coagulation cascade, fibrinogen is cleaved by thrombin to generate fibrin monomers, which spontaneously polymerize into protofibrils. The fibrin protofibrils then aggregate into larger branching fibers that form a 3D network providing the primary barrier to prevent loss of erythrocytes during the initial stages of hemostasis. Depending on the stoichiometry of fibrinogen and thrombin, and other local factors, the structure of the fibrin fiber network may be relatively porous (composed of thick, loosely packed fibers) or dense (composed of thin, tightly packed fibers) (8). Additional protection against permeability is provided by platelet retraction, leading to contraction of the fibrin- and platelet-containing clot during late hemostasis and early wound healing.

It has been recognized for decades that fibrin can limit bacterial invasion and dissemination (9). In mice, fibrinogen

Related Article: p. 3356

deficiency (6) or expression of a mutant form of fibrinogen that lacks the ability to form fibrin (7) results in impaired microbial clearance and decreased survival after infection with certain bacterial pathogens. Fibrin-mediated host antimicrobial activity is thought to occur through two general mechanisms: (a) the fibrin matrix functions as a direct protective barrier in microbial invasion; and (b) fibrin and/or its precursor fibrinogen binds to and activates innate immune cells, such as neutrophils, at sites of injury (3). Not surprisingly, some pathogenic bacteria have evolved the capacity to co-opt the fibrin matrix barrier to evade innate immune surveillance (6). For example, Staphylococcus aureus can directly bind to fibrin fibers, leading to the formation of biofilms, bacterial persistence, abscesses, and colonization of indwelling medical devices $(10,11)$. Thus, depending on the context, fibrin can support either microbial virulence or antimicrobial defense.

\section{Structure of the external face of the fibrin clot}

In this issue (12), Macrae and colleagues provide evidence that a previously overlooked fibrin structure may play a role in initial microbial defense. Using scanning electron microscopy to investigate the structural characteristics of fibrin clots formed at the air-liquid interface in vitro, the authors observed that fibrin at the clot surface is not arranged in the form of conventional polymeric fibers but instead forms a continuous 2D sheet that covers the exterior face of the clot. Similar fibrin films were also observed on the external surface of fibrin clots formed in vivo in a mouse cutaneous injury model. Further characterization of the fibrin films demonstrated that they are composed primarily of fibrin/fibrinogen and can be lysed by plasmin. Fibrin film formation does not appear to require platelets or fibrin crosslinking by factor XIII and can be prevented by surfactants. 


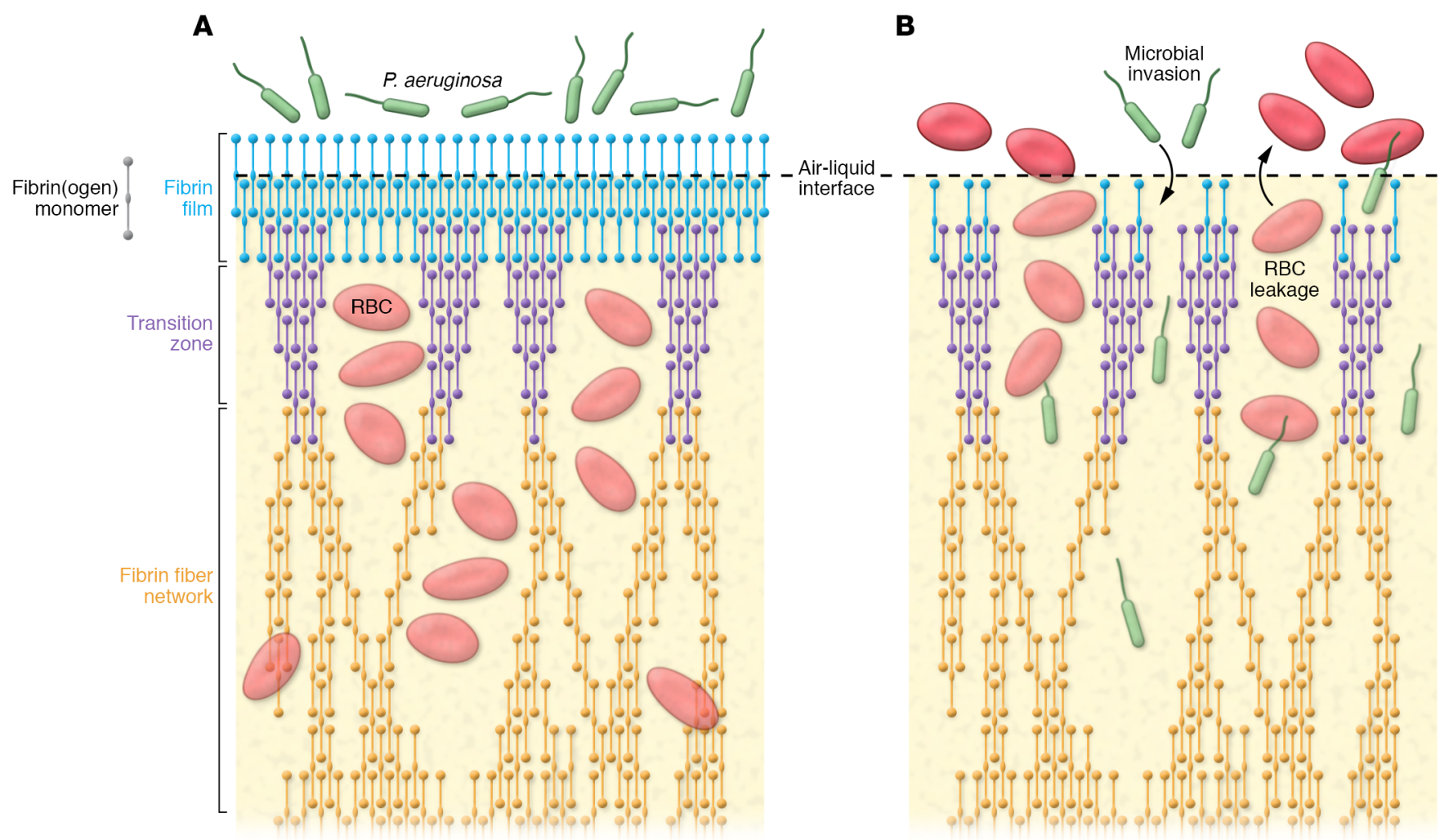

Figure 1. Structure and function of fibrin films. (A) Schematic representation of a film composed of fibrinogen and fibrin monomers forming a continuous $2 \mathrm{D}$ sheet at the air-liquid interface. Tethering fibrin protofibrils extend through a transition zone into a network of branching polymeric fibrin fibers. The fibrin film functions as a barrier at the external clot surface to RBC and prevents microbial invasion into the wound. (B) Disruption of the fibrin film allows infiltration of microbes such as Pseudomonas aeruginosa and leakage of RBC. Blue: fibrin D domain; red, E domain.

Due to its so-called knob-hole polymerization mechanism, fibrin can form both fibers and films (13), but the potential biological importance of fibrin films has been largely overlooked. Macrae and coauthors propose a model in which, upon cutaneous injury, fibrinogen molecules rapidly form an organized monolayer at the air-liquid interface through a biophysical mechanism similar to the formation of Langmuir films by amphipathic molecules (14). As hemostatic pathways are activated and thrombin begins to generate fibrin, fibrin monomers interdigitate with fibrinogen within the film, forming a thin, tightly packed, continuous sheet of fibrin(ogen) oriented perpendicularly to the air-liquid interface. The surface sheet connects, through tethering fibrin protofibrils, to the nascent fibrin fiber network deeper within the clot (Figure 1A). These findings clarify the integrated architecture of fibrin formed at the external face of blood clots and provide insight into the long-standing mystery of how fibrin fiber networks are formed without leaving free ends at the clot boundaries (13).

\section{Biological significance and clinical implications of fibrin films}

The observations of Macrae et al. suggest that in response to cutaneous injury, the fibrin film forms an instant barrier that constitutes an initial line of host defense against microbial infiltration while allowing the underlying clot to mature and more fully engage the innate immune system. In agreement with this model, fibrin films tended to form more readily with low concentrations of thrombin, as might be expected in the very early stages of hemostasis. To test the hypothesis that fibrin films function as bioprotective barriers, Macrae et al. utilized a Boyden chamber to examine the migration of three types of bacteria commonly found in skin flora (Escherichia coli, Staphylococcus epidermidis, and Staphylococcus aureus). In each case, when the fibrin film was intact, little to no bacterial infiltration through the clot was observed within the first 36 hours. In contrast, when the fibrin film was disrupted by a surfactant, bacte- rial infiltration occurred markedly sooner (within 24 hours). Disruption of the fibrin film also resulted in increased leakage of RBC (Figure 1B). Importantly, the protective barrier effect was confirmed in a murine model of dermal infection with Pseudomonas aeruginosa.

The article by Macrae et al. is interesting because it sheds new light on a previously overlooked aspect of fibrin structural biology and also because it suggests a novel mechanism for fibrin-mediated collaboration between the hemostatic and innate immune systems. This study also raises questions about the potential clinical implications of fibrin films for microbial host defense. Macrae and colleagues suggest that the barrier function of fibrin films may play an important role in controlling infection at sites of cutaneous injury, an idea that is perhaps supported by the high prevalence of tenacious dermal infections in afibrinogenemia. However, there are many other immune mechanisms to limit microbial invasion, and the relative importance of fibrin films is uncertain. Is this simply a 
curiosity of fibrin polymer structure or a clinically important pathophysiological process? It remains to be determined whether wound treatments that specifically disrupt fibrin film formation lead to an increase in infection morbidity or mortality, or if engineered fibrin films can be developed to reduce local infection after injuries.

Although the article by Macrae et al. focuses entirely on fibrin films formed at the air-liquid interface, it is conceivable that fibrin may also form Langmuir-like films on other surfaces, such as stents, medical devices, or even natural surfaces at internal sites of vascular injury. If this is the case, fibrin films might function as nucleation sites for the initiation of intravascular fibrin fiber growth in thrombotic conditions associated with low-grade thrombin generation, such as disturbed arterial blood flow. Finally, fibrin films might tether fibrin-rich thrombi to native or artificial vascular surfaces and influence the risk of thromboembolism. Hopefully, the Macrae article will stimulate future investigation into these and other interesting questions.

\section{Acknowledgments}

This work was supported by the National Heart, Lung and Blood Institute (T32 HL007344 and R01 HL118742).

Address correspondence to: Steven R. Lentz, Department of Internal Medicine, C21 GH, The University of Iowa Carver College of Medicine, 200 Hawkins Drive, Iowa City, Iowa 52242, USA. Phone: 319.356.4048;

Email: steven-lentz@uiowa.edu.

1. Engelmann B, Massberg S. Thrombosis as an intravascular effector of innate immunity. Nat Rev Immunol. 2013;13(1):34-45.

2. Esmon CT. Interactions between the innate immune and blood coagulation systems. Trends Immunol. 2004;25(10):536-542.

3. Foley JH, Conway EM. Cross talk pathways between coagulation and inflammation. Circ Res. 2016;118(9):1392-1408.

4. Oehmcke-Hecht S, Köhler J. Interaction of the human contact system with pathogens an update. Front Immunol. 2018;9:312.

5. Maas C, Renné T. Coagulation factor XII in thrombosis and inflammation. Blood. 2018;131(17):1903-1909.

6. Degen JL, Bugge TH, Goguen JD. Fibrin and fibrinolysis in infection and host defense. J Thromb Haemost. 2007;5(suppl 1):24-31.
7. Prasad JM, et al. Mice expressing a mutant form of fibrinogen that cannot support fibrin formation exhibit compromised antimicrobial host defense. Blood. 2015;126(17):2047-2058.

8. Undas A, Ariëns RA. Fibrin clot structure and function: a role in the pathophysiology of arterial and venous thromboembolic diseases. Arterioscler Thromb Vasc Biol. 2011;31(12):e88-e99.

9. Zinsser HH, Pryde AW. Experimental study of physical factors, including fibrin formation, influencing the spread of fluids and small particles within and from the peritoneal cavity of the dog. Ann Surg. 1952;136(5):818-827.

10. Ko YP, Flick MJ. Fibrinogen is at the interface of host defense and pathogen virulence in Staphylococcus aureus infection. Semin Thromb Hemost. 2016;42(4):408-421.

11. McAdow M, Missiakas DM, Schneewind O. Staphylococcus aureus secretes coagulase and von Willebrand factor binding protein to modify the coagulation cascade and establish host infections. J Innate Immun. 2012;4(2):141-148.

12. Macrae FL, et al. A fibrin biofilm covers blood clots and protects from microbial invasion. J Clin Invest. 2018;128(8):3356-3368.

13. O'Brien ET, Falvo MR, Millard D, Eastwood B, Taylor RM, Superfine R. Ultrathin selfassembled fibrin sheets. Proc Natl Acad Sci U S A. 2008;105(49):19438-19443.

14. Zasadzinski JA, Viswanathan R, Madsen L, Garnaes J, Schwartz DK. Langmuir-Blodgett films. Science. 1994;263(5154):1726-1733. 